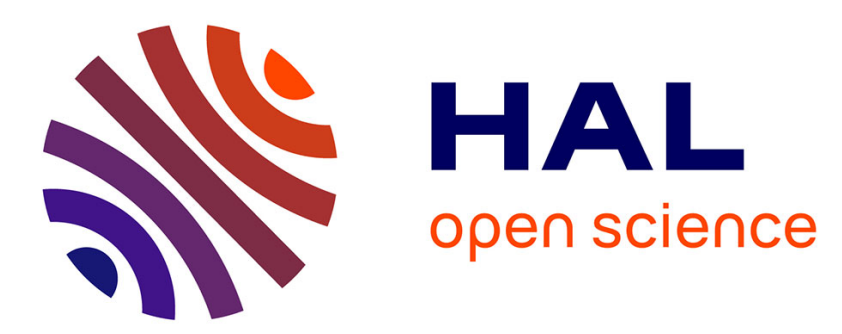

\title{
A Reference Governor approach for Lateral Control of Autonomous Vehicles
}

\author{
Dimitrios Kapsalis, Olivier Sename, Vicente Milanes, John Jairo Martinez
} Molina

\section{- To cite this version:}

Dimitrios Kapsalis, Olivier Sename, Vicente Milanes, John Jairo Martinez Molina. A Reference Governor approach for Lateral Control of Autonomous Vehicles. 24th IEEE International Conference on Intelligent Transportation - ITSC2021, Sep 2021, Indianapolis, United States. hal-03280581v1

\section{HAL Id: hal-03280581 \\ https://hal.univ-grenoble-alpes.fr/hal-03280581v1}

Submitted on 7 Jul 2021 (v1), last revised 22 Jul 2021 (v2)

HAL is a multi-disciplinary open access archive for the deposit and dissemination of scientific research documents, whether they are published or not. The documents may come from teaching and research institutions in France or abroad, or from public or private research centers.
L'archive ouverte pluridisciplinaire HAL, est destinée au dépôt et à la diffusion de documents scientifiques de niveau recherche, publiés ou non, émanant des établissements d'enseignement et de recherche français ou étrangers, des laboratoires publics ou privés. 


\title{
A Reference Governor approach for Lateral Control of Autonomous Vehicles
}

\author{
Dimitrios Kapsalis ${ }^{1,2}$, Olivier Sename ${ }^{2}$, Vicente Milanes ${ }^{1}$ and John J. Martinez ${ }^{2}$
}

\begin{abstract}
This paper presents a novel approach on the union of lane tracking/change via the use of a Reference Governor (RG). This is achieved by feeding a modification of the reference during lane-change, called "virtual" reference, to the predesigned lane-tracking controller. The suggested methodology ensures reference is smooth enough so that the tracking controller does not overshoot and sustains fast convergence to the adjacent lane. The virtual reference is obtained as the solution of a convex optimization problem so that future closedloop states are enforced to belong in an admissible set. The efficiency of the proposed control-scheme is illustrated in highfidelity simulation results, for the case of an automated Renault Zoe. Consequently, the lane-tracking controller, a $H_{\infty}$ yaw-rate tracker for which robustness certificates are presented, is able to perform a lane change maneuver when the RG is used.
\end{abstract}

\section{INTRODUCTION}

Autonomous vehicles must be able to perform different tasks and simultaneously sustain high performance and comfort for the passengers. Lateral control plays a key role to achieve those tasks and it is often separated into lane tracking and lane change maneuvers [1].

Most of the developed lane-tracking control algorithms are based on vision dynamics aiming to reach a target point at a look-ahead distance in front of the vehicle with respect to the reference trajectory, minimizing either (i) the corresponding lateral error [2]; or (ii) the yaw-rate error by calculating the curvature of the vehicle trajectory in the target point [3]. [4] considers the global coordinates of a point onto the trajectory as set points, where the distance from the vehicle's position has to be minimized. From the control aspect, lane change maneuvers can be treated as a lane-tracking scenario where an adjacent lane is treated as the reference path [5]. Consequently, the aforementioned techniques can be used to perform lane tracking and lane change maneuvers as well.

On the other hand, governors are add-on schemes that emerged as a way to treat constraints for a pre-designed closed-loop system [6]. The objective is to produce a virtual reference, for which saturation constraints or even performance criteria are satisfied. The RG algorithm emerged for discrete time systems. In [7], a dynamic update formula of the virtual reference was proposed, taking the previous value of reference in the optimization problem. From the

\footnotetext{
${ }^{1}$ Dimirios Kapsalis and Vicente Milanes are with the Research Department, Renault SAS, 1 Avenue de Golf, 78280 Guyancourt, France dimitrios.kapsaliserenault.com vicente.milaneserenault.com

${ }^{2}$ Dimitrios Kapsalis, John Jairo Martinez Molina and Olivier Sename are with Univ. Grenoble Alpes, CNRS, Grenoble INP, GIPSA-Lab, 38000 Grenoble, France \{dimitrios.kapsalis, john-jairo.martinez-molina, olivier.sename\}agrenoble-inp.fr
}

computational aspect, the virtual reference is computed online as the maximal one for which the closed-loop trajectories stay in an approximation of the maximal output admissible set [8]. Furthermore, command governor algorithms consider the virtual reference explicitly included in the closed-loop system without any parameterization [9]. In that case, the virtual reference is found as the solution of the on-line minimization of the quadratic euclidean distance between the virtual and the desired reference.

\section{A. Related Works}

Several governor approaches have been applied and implemented successfully in automotive systems [10]. In [11] the vehicle rollover avoidance is tackled modifying the driver steering input with an active steering system. In [12] a distributed RG approach is proposed to ensure the string stability and energy efficiency in a cooperative adaptive cruise control scheme.

\section{B. Motivations and contributions}

This paper presents the design of a control system that enables a single lane-tracking controller to handle adequately the case of a lane change as well. In that sense, it can be avoided the need to design a second controller that performs only during the lane-change and even the switching scheme between these two controllers [13].

For the sake of clarity, Fig. 1 illustrates two real yaw-rate references recovered in experimental tests using a Renault Zoe in autonomous mode. The vehicle starting in a straight line, for fixed velocity $v_{x}=10 \mathrm{~m} / \mathrm{s}$, (i) in blue it followed a turn (lane tracking) and (ii) in red performed a fast maneuver to reach an adjacent lane more than 3 meters away from the initial position of the vehicle (lane-change). As it can been seen, the yaw-rate reference during lane-tracking is gradually increasing and, in general, is smooth without abrupt changes. On the other hand, at the beginning of a lane-change the yaw-rate reference is increased discontinuously, function of the required lateral displacement of the car. Then, as the vehicle approaches the desired lane, the yaw-rate reference gradually reduces till zero where the maneuver is performed. Therefore, to treat the discontinuous phase, usually a lanechange controller has restricted capabilities (compared to a lane-tracking one) so as not to go further the steering rate limitations of the actuator and cause oscillations or even instability [13].

To address the aforementioned problem, the $\mathrm{RG}$ is an interesting solution to guarantee performances in both cases while handling the actuator limitations. 


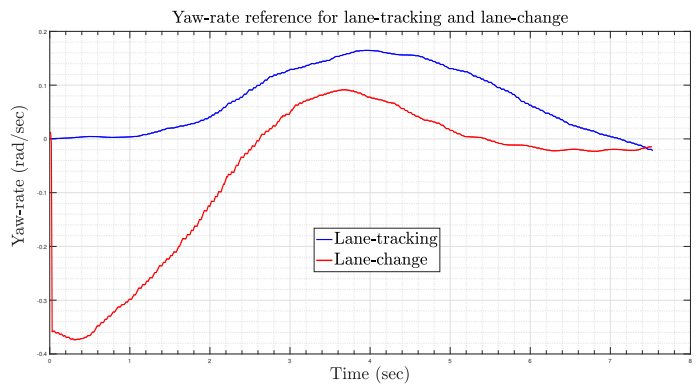

Fig. 1. Yaw-rate references for the case of tracking a turn (blue) and for a lane-change maneuver (red) recovered in experimental tests while driving in autonomous mode an automated Renault Zoe for $v_{x}=10 \mathrm{~m} / \mathrm{s}$.

The main novelties of this paper are summarized below:

- The union of lane-change and lane-tracking scenarios is achieved by using a yaw-rate tracking controller equipped with a RG. During lane-change, a virtual yaw-rate reference that is smooth is generated, setting feasible the yaw-rate tracker to follow it.

- The selected steering limit is provided as an extra degree of freedom in the design procedure.

- The imminent objectives are satisfied by a real-time optimization problem, fast enough for implementation.

\section{Structure of the Paper}

The rest of the paper is structured as follows. Section II presents the lateral control framework where the robust $H_{\infty}$ steering controller is based-on. Section III includes an overview of the dynamic RG design for linear systems and the design for the lane-tracking system. Section IV shows the simulation results for the proposed methodology proving that the governed lane-tracking controller performs a lane-change maneuver for different steering wheel angle bounds.

\section{LATERAl Control Framework FOR the SteERING CONTROLlER DESIGN}

This section presents the bicycle model used for the design of the $H_{\infty}$ lane-tracking controller. An analysis on the frequency domain is illustrated to prove the robustness of the closed-loop system when different parameters of the vehicle model are considered uncertain.

\section{A. Model Formulation}

The lateral motion of the vehicle is expressed as a bicycle model [14], which is depicted in Fig. 2. $\alpha_{f}, \alpha_{r}$ are the tire side slip angles of the front and rear wheels respectively. $\beta$ is the side slip angle of the vehicle. $L_{f}, L_{r}$ are the distances of the front and rear wheel with respect to the center of the gravity of the car. $C_{f}, C_{r}$ the front and rear cornering stiffness as a result from the linear approximation of the lateral forces applied on the tires, i.e $F_{y f}=C_{f} \alpha_{f}, F_{y r}=C_{r} \alpha_{r} . m$ is the mass and $I_{z}$ the car inertia.

Assuming the longitudinal velocity of the vehicle as an exogenous parameter, considered measured at every instant, a linear model can be formulated with states the lateral speed $v_{y}$ and the yaw-rate $\dot{\psi}$ at the center of gravity.

$$
\begin{aligned}
& \dot{x}(t)=A x(t)+B u(t) \\
& y(t)=C x(t)+D u(t)
\end{aligned}, G=\left[\begin{array}{l|l}
A & B \\
\hline C & D
\end{array}\right]
$$

with, $A=\left[\begin{array}{cc}-\frac{C_{f}+C_{r}}{m v_{x}} & -v_{x}+\frac{C_{r} L_{r}-C_{f} L_{f}}{m v_{x}} \\ \frac{-L_{f} C_{f}+L_{r} C_{r}}{I_{z} v_{x}} & -\frac{L_{f}^{2} C_{f}+L_{r}^{2} C_{r}}{I_{z} v_{x}}\end{array}\right]$,
$B=\left[\begin{array}{c}\frac{C_{f}}{m} \\ \frac{L_{f} C_{f}}{I_{z}}\end{array}\right], C=\left[\begin{array}{ll}0 & 1\end{array}\right], D=0$.

The state space vector is $x(t)=\left[\begin{array}{c}v_{y} \\ \dot{\psi}\end{array}\right]$ and the input $u(t)=\delta$ is the steering wheel angle.

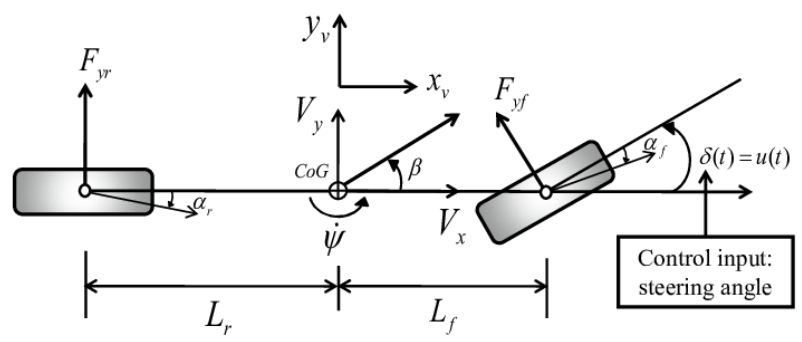

Fig. 2. Two-wheeled bicycle model

Moreover, so as to have a more realistic model for the steering of the vehicle, a second-order identified transfer function is used as the actuator model as such:

$$
G_{a c t}=\frac{k}{s^{2}+2 j w_{n} s+w_{n}^{2}} e^{-T_{d} s}
$$

where $k$ is the static gain, $w_{n}$ the natural frequency, $j$ the damping and $T_{d}$ the time-delay of the actuator model.

This model is connected in series with the bicycle model $G$ (1) in order to formulate an even more realistic model of the vehicle $G_{v}$ including the actuator limitations $G_{a c t}$ i.e, $G_{v}=G_{a c t} G$.

\section{B. $H_{\infty}$ Dynamic Output Controller Design}

The control algorithm is based on tracking a yaw-rate reference $r(t)$, which is computed in order to reach a target point located on a look-ahead distance onto the reference trajectory [3]. The lane-following system has to perform fast tracking capabilities and provide comfort as well.

The lane-tracking controller is designed, as it is shown in Fig. 3, using the $H_{\infty}$ method where the performance is achieved by the weighting functions $W_{e}(s)$ and $W_{u}(s)$ [15]. The first-order filters $W_{u}(s)$ and $W_{e}(s)$ are chosen as:

- $W_{u}(s)=\frac{s+\omega_{u} / M_{u}}{\varepsilon_{u} s+\omega_{u}}$, where $\omega_{u}=10 \mathrm{rad} / \mathrm{s}$ is the bandwidth of the controller and have a smooth steering wheel angle change. $M_{u}=2(6 d b)$ is chosen in order 


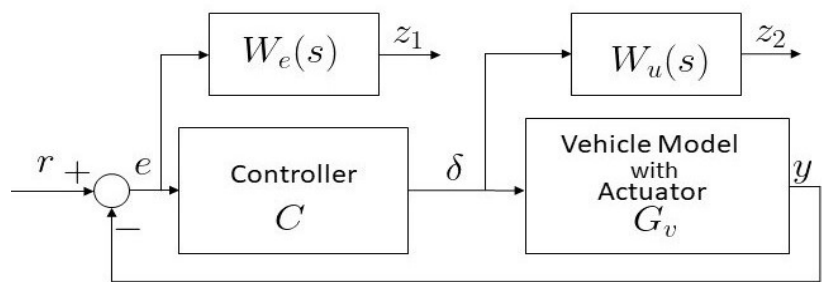

Fig. 3. Lateral control design via yaw-rate tracking.

to satisfy the saturation limits of the actuator and $\varepsilon_{u}=0.1$ which is the roll-off frequency for better noise attenuation.

- $W_{e}(s)=\frac{s+\omega_{e} / M_{e}}{\varepsilon_{e} s+\omega_{e}}$, where $\omega_{e}=3 \mathrm{rad} / \mathrm{s}$ is the bandwidth for fast tracking of the reference. $M_{e}=2(6 \mathrm{db})$ is chosen to ensure robustness and $\varepsilon_{e}=0.001$ which corresponds to the steady state tracking error.

The tuning of the dynamic output controller is achieved by minimizing off-line the performance variables $z=\left[\begin{array}{l}z_{1} \\ z_{2}\end{array}\right]$ with respect to the reference $r$ for a performance index $\gamma>0$, i.e

$$
\sup _{\|r\| \neq 0} \frac{\|z\|_{2}}{\|r\|_{2}}<\gamma
$$

The design of the nominal $H_{\infty}$ controller is carried out assuming a constant longitudinal speed $v_{x}=10 \mathrm{~m} / \mathrm{s}$. Solving the appropriate linear matrix inequalities [16] leads to the optimal attenuation level $\gamma=0.9053$. Since $\gamma<1$, the closedloop met the required objectives for the nominal case where $v_{x}=10 \mathrm{~m} / \mathrm{s}$ (see Fig 4).

A first robustness analysis is presented in Fig. 5, where are shown the frequency responses of the performance variables $z$ to the reference $r$ (so the $S$ and $K S$ sensitivity functions) relatively to the specifications $1 / W_{e}, 1 / W_{u}$ imposed, considering an uncertain velocity i.e $v_{x} \in[5,15]$. This emphasizes the robust performance of the closed-loop system w.r.t speed uncertainties.
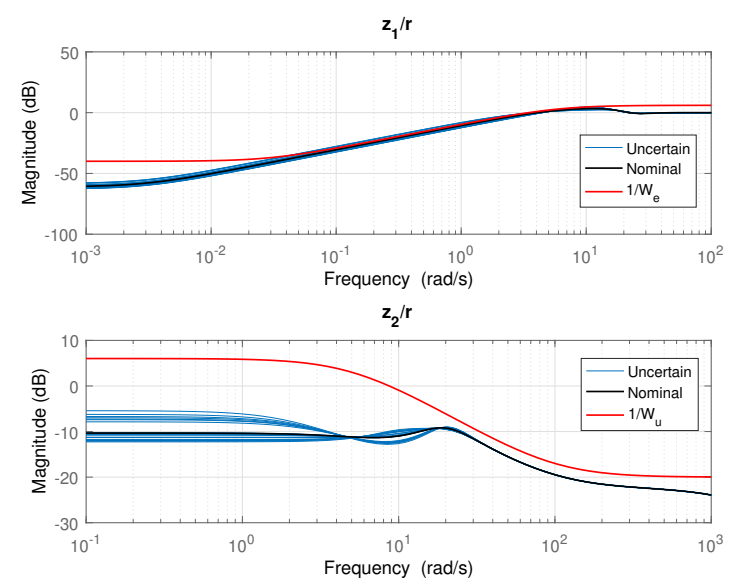

Fig. 4. Frequency responses $z_{1} / r$ and $z_{2} / r$ of the closed-loop system.
Note that the dynamic output controller can be expressed in a state space form, which has as input the yaw-rate error $e(t)=r(t)-y(t)$ and as an output the steering wheel angle $\delta(t)$.

$$
\begin{gathered}
\dot{x}_{c}(t)=A_{c} x_{c}(t)+B_{c} e(t) \\
\delta(t)=C_{c} x_{c}(t)+D_{c} e(t)
\end{gathered}, C=\left[\begin{array}{l|l}
A_{c} & B_{c} \\
\hline C_{c} & D_{c}
\end{array}\right]
$$

where, $x_{c}(t) \in \mathbb{R}^{8}$ is the state vector of the controller, $A_{c} \in$ $\mathbb{R}^{8 \times 8}, B_{c} \in \mathbb{R}^{8}$ and $C_{c} \in \mathbb{R}^{1 \times 8}$ are the controller's state space matrices.

C. Robust stability and performance analysis using $\mu$ analysis

In this section, a more complete robustness analysis is carried out considering several parameter uncertainties.

First let us recall that a control system is robust if it is insensitive to differences between the actual real system and the model used to design the controller. This is formulated through the well know concepts below (see [15]):

- Robust Stability (RS). The system is stable for all perturbed plants about the nominal model up to the worst-case model uncertainty.

- Robust Performance (RP). The system satisfies the performance specifications for all perturbed plants about the nominal model up to the worst-case model uncertainty.

Now, for a deeper analysis, the $\mu$-analysis methodology is considered to conclude on the robustness of the proposed $H_{\infty}$ controller w.r.t parameter uncertainties (see [15]). We here consider that the parameters of the bicycle model are uncertain as given below

$$
\begin{array}{llll}
\overline{C_{f}}=C_{f}\left(1+p_{C_{f}} \delta_{C_{f}}\right), & p_{C_{f}}=15 \%, & \delta_{C_{f}} \in[-1,1] \\
\overline{C_{r}}=C_{r}\left(1+p_{C_{r}} \delta_{C_{r}}\right), & p_{C_{r}}=15 \%, & \delta_{C_{r}} \in[-1,1] \\
\bar{m}=m\left(1+p_{m} \delta_{m}\right), & p_{m}=10 \%, & \delta_{m} \in[-1,1] \\
\overline{I_{z}}=I_{z}\left(1+p_{I_{z}} \delta_{I_{z}}\right), & & p_{I_{z}}=10 \%, & \delta_{I_{z}} \in[-1,1]
\end{array}
$$

As we consider structured uncertainties, a $\mu$-analysis is used to study RS and RP. The method consists in applying the $\mu$ small gain theorem for structured uncertainties, so to build the so-called $N-\Delta$ form representing the uncertain closedloop system. In this framework, $N$ is written as $N(s)=$ $\left[\begin{array}{ll}N_{11}(s) & N_{12}(s) \\ N_{21}(s) & N_{22}(s)\end{array}\right]$, and the closed-loop transfer matrix is:

$$
T_{e w}(s)=N_{22}(s)+N_{21}(s) \Delta(s)\left(I-N_{11}(s)\right)^{-1} N_{12}(s)
$$

First the structured singular value is defined as:

$$
\mu_{\underline{\Delta}}(M)^{-1}:=\min \{\bar{\sigma}(\Delta): \Delta \in \underline{\Delta}, \operatorname{det}(I-\Delta M) \neq 0\} .
$$

Assuming nominal stability, RS and RP analysis for structured uncertainties are therefore such that:

$$
\begin{aligned}
& \mathrm{RS} \Leftrightarrow \mu_{\underline{\Delta}}\left(N_{11}\right)<1, \forall \omega \\
& \mathrm{RP} \Leftrightarrow \mu_{\underline{\Delta}}(N)<1, \forall \omega
\end{aligned}
$$

Finally, let us remark that the structured singular value cannot be explicitly determined, so that the method consists in 
calculating an upper bound and a lower bound, as closed as possible to $\mu$.

Now as shown below the maximal bounds of the $\mu$ for RS and RP are less than one, then the $H_{\infty}$ controller keeps stability and performance for the considered uncertainties.
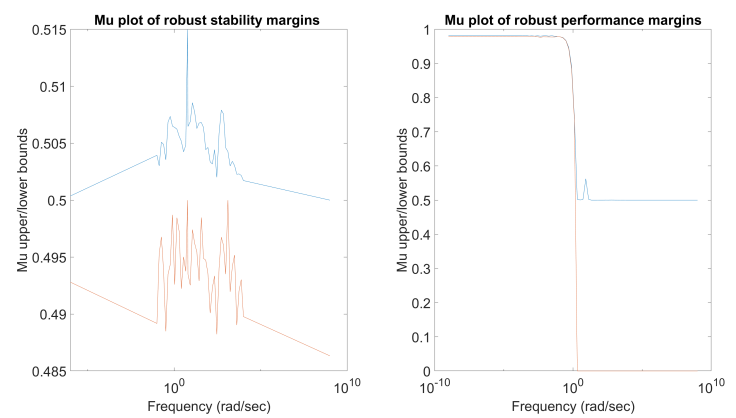

Fig. 5. Mu bounds of the Robust stability and Robust Performance analysis

\section{REFERENCE GOVERNOR DESIGN FOR THE Closed-Loop System}

The first part of this section presents the needed background on RG for this study. Then, the design of the proposed RG for the lane-keeping system is detailed in order to perform a lane-change maneuver.

\section{A. Brief Background On Reference Governors For Linear Systems}

The RG is enforced to discrete-time closed-loop systems whose state space dynamics are expressed as:

$$
\begin{gathered}
x_{c l}(k+1)=A x_{c l}(k)+B v(k) \\
y_{c l}(k)=C x_{c l}(k)+D v(k) \in Y
\end{gathered}
$$

where $x_{c l} \in R^{n}$ is the state space vector, $v \in R^{m}$ is the virtual reference, $y_{c l} \in R^{p}$ is the output vector and $Y \subseteq R^{p}$ is the constraint output admissible set.

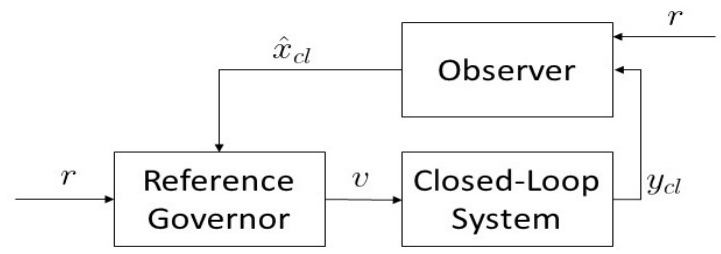

Fig. 6. Full control-scheme including the RG

The scheme in Fig. 6 depicts the general structure of the RG. An initial reference $r \in R^{q}$ is fed to the RG and assuming full information of the state or its estimation $\hat{x}_{c l}, \mathrm{RG}$ alters it to the virtual reference $v$. The alteration is made in order to satisfy closed-loop constraints, keeping $v$ close to $r$.

The fulfilment of the constraints is achieved by

$$
\left(v, \hat{x}_{c l}\right) \in O_{\infty}
$$

where $O_{\infty} \subseteq R^{m} \times R^{n}$. The set $O_{\infty}$ is the maximal output admissible set which contains the set of all states $\hat{x}_{c l}$ and constant references $v$ for which constraints are satisfied for present and future time $k$, i.e

$$
O_{\infty}=\left\{\left(v, \hat{x}_{c l}\right): y_{c l}(k) \in Y, \forall k \in Z_{+}\right\}
$$

where the output $y(k)$ at every time instant $k$ can be computed as follows:

$$
y_{c l}(k)=C A^{k} \hat{x}_{c l}+C\left(I-A^{k}\right)(I-A)^{-1} B v+D v
$$

To ensure robustness and steady state constraints enforcement, $O_{\infty}$ is tightened as:

$$
\begin{gathered}
\tilde{O}_{\infty}=O_{\infty} \cap O^{\varepsilon}, \text { with } \\
O^{\varepsilon}=\left\{v: \bar{y}_{v, c l} \in(1-\varepsilon) Y\right\}, 1 \gg \varepsilon>0
\end{gathered}
$$

where the steady state output response $\bar{y}_{v}$ is expressed as such:

$$
\bar{y}_{v, c l}=C(I-A)^{-1} B v+D v
$$

If $A$ is Schur and the pair $(C, A)$ is observable, it is proven in [8] that $\tilde{O}_{\infty}$ is finitely determined, i.e there exists a finite time index $k^{*}$ such that:

$$
\tilde{O}_{\infty}=\left\{\left(v, \hat{x}_{c l}\right): y_{c l}(k) \in Y, k=0, . ., k^{*}\right\} \cap O^{\varepsilon}
$$

In the simplest case, where the constraint set $Y$ is expressed in a polytopic form, i.e as a set of linear inequalities as explained below:

$$
Y=\left\{y: H y_{c l}(k) \preceq h, \forall k \in Z_{+}\right\}, h>0
$$

then, the computation of the $\tilde{O}_{\infty}$ is easy. More precisely, given a $1 \gg \varepsilon>0$ and sufficiently large $k^{*}$,

$\tilde{O}_{\infty}=\left\{\left(v, \hat{x}_{c l}\right):\left[\begin{array}{cc}H D & H C \\ H C B+H D & H C A \\ \vdots & \vdots \\ H C\left(I-A^{k^{*}}\right)(I-A)^{-1} B+H D & H C A^{k^{*}} \\ H C(I-A)^{-1} B+H D & 0\end{array}\right]\left[\begin{array}{c}v \\ \hat{x}_{c l}\end{array}\right] \preceq\left[\begin{array}{c}h \\ h \\ \vdots \\ h \\ (1-\varepsilon) h\end{array}\right]\right\}$

Finally, a minimal representation of $\tilde{O}_{\infty}$ is obtained when redundant or almost redundant inequality constraints are eliminated. The reduced inequalities of (17) that express the maximal admissible set are written as:

$$
\tilde{O}_{\infty}=\left\{\left(v, \hat{x}_{c l}\right): \mathscr{H}_{v} v+\mathscr{H}_{x} \hat{x}_{c l} \preceq \mathscr{L}\right\}
$$

where $\mathscr{H}_{v}, \mathscr{H}_{x}$ and $\mathscr{L}$ are the matrices that correspond to the reduced inequalities.

According to the dynamic $\mathrm{RG}$, at every instant $k$, a line search along the segment $v(k-1)$ and $r(k)$ is performed in the convex set $\tilde{O}_{\infty}$ so as to compute the virtual reference solving the following optimization problem:

$$
\begin{array}{cl}
\underset{K \in[0,1]}{\operatorname{maximize}} & K \\
\text { subject to } & v(k)=v(k-1)+K(r(k)-v(k-1)) \\
& \mathscr{H}_{v} v(k)+\mathscr{H}_{x} \hat{x}_{c l}(k) \preceq \mathscr{L}
\end{array}
$$




\section{B. Reference Governor Design for Lane-Change Maneuvers}

The following subsection describes the design of the RG for lateral control. The aim of the $\mathrm{RG}$ is, given a lanetracking controller, 1) to generate a smooth virtual reference that permits the lane-change maneuver and 2) respects the maximum steering actuation capabilities.

The first step of the design of the RG is the selection of the model used for prediction, which in this paper is chosen as the closed-loop system between the vehicle model $G$ and the controller $C$, eq. (2) and (4) respectively. This model is discretized at the sampling time $T_{s}=0.01 \mathrm{~s}$, for which the automated vehicle accepts steering command. The discrete state space model is expressed below:

$$
\begin{gathered}
x_{c l}(k+1)=A_{d} x_{c l}(k)+B_{d} r(k) \\
y_{c l}(k+1)=C_{d} x_{c l}(k)+D_{d} r(k) \in Y
\end{gathered}
$$

where $x_{c l}=\left[\frac{x}{x_{c}}\right] \in \mathbb{R}^{10}$ is the closed-loop state space vector (without the states of the actuator so as to reduce the size of the optimization), $y_{c l}=\delta$ is the steering wheel angle enforced to stay in the constraint admissible set $Y, A_{d} \in$ $\mathbb{R}^{10 \times 10}, B_{d} \in \mathbb{R}^{10}, C_{d}=\left[0 \mid C_{c}\right] \in \mathbb{R}^{1 \times 10}, D_{d}=0$ are the closed-loop system space matrices.

To implement the control scheme depicted in Fig. 6, an LTI observer has been designed so as to provide full knowledge over the state $x_{c l}$ at every instant for the system (20). Moreover, the constraint admissible set is constructed to include boundaries of the steering angle.

$$
-\delta_{\max } \leq y_{c l}(k) \leq \delta_{\max }
$$

where $\delta_{\max }$ is the maximum admissible steering angle in rad. $Y$ can be written in a polytopic form below:

$$
Y=\left\{y_{c l}: H y_{c l}(k) \leq h, \forall k \in Z_{+}\right\}, h>0
$$

where $H=\left[\begin{array}{cc}-1 & 0 \\ 0 & 1\end{array}\right], h=\left[\begin{array}{c}\delta_{\max } \\ \delta_{\max }\end{array}\right], \delta_{\max }=\frac{\pi}{180 \kappa} \delta_{\text {max }}^{\circ}>0$, $\delta_{\text {max }}^{\circ}$ is the selected maximum value in degrees and the gain $\kappa$ is the gear ratio of the steering actuation system.

The objective of rendering smooth the virtual yaw-rate reference, feasible for the lane-tracking controller, is chosen as an extra constraint which limits the slew rate of the virtual reference as follows:

$$
|v(k)-v(k-1)| \leq \Delta_{v}
$$

where $\Delta_{v}$ is the selected slew rate of the virtual reference.

That constraint is included in the optimization problem (19). This leads to the proposed maximization from which the virtual reference $v$ is found on-line as such:

$$
\begin{array}{cl}
\underset{K \in[0,1]}{\operatorname{maximize}} & K \\
\text { subject to } & v(k)=v(k-1)+K(r(k)-v(k-1)) \\
& \mathscr{H}_{v} v(k)+\mathscr{H}_{x} \hat{x}_{c l}(k) \preceq \mathscr{L} \\
& |v(k)-v(k-1)| \leq \Delta_{v}
\end{array}
$$

The set $\tilde{O}_{\infty}$ has been computed off-line, for $\varepsilon=10^{-6}, k^{*}=7$ for the two different cases where $\delta_{\max }^{\circ}=50^{\circ}, 100^{\circ}$, and its minimal representation matrices $\mathscr{H}_{v}, \mathscr{H}_{x}$ and $\mathscr{L}$ are obtained using Multi-Parametric Toolbox [17]. The slew rate of the virtual reference $\Delta_{v}$ is selected as 0.01 to enable a smooth lane-change maneuver when the $H_{\infty}$ lane-tracking controller is present. The embedded solver used for the maximization problem (24), that computes $v$ on-line, is CVXGEN [18].

\section{Simulation Results}

The parameters of the vehicle model in (1) correspond to an automated Renault Zoe (see [2] for details). We consider as a case scenario where the vehicle starts more than 3 meters away from the reference lane and simulate closer to the collected experimental data. In that way, the lane-keeping system equipped with the RG can prove that performs a lanechange maneuver. The speed is fixed at $v_{x}=10 \mathrm{~m} / \mathrm{s}$.

Fig. 7 illustrates the lateral deviation during the lanechange scenario. When the $\mathrm{RG}$ is used, a smooth convergence is achieved to the reference lane with maximum overshoot of $0.1 \mathrm{~m}$. When the steering wheel angle bound is $100^{\circ}$, the convergence is faster than $50^{\circ}$. On the contrary, when the RG is absent the lane-tracking controller shows jerky performance since it oscillated before converging to the desired lane.

Fig. 8 depicts the steering wheel angles during the maneuver for all cases. When the RG is added to the loop, the controller's output is smooth without violating the imposed constraint for $\delta_{\text {max }}=50^{\circ}$. As for case where $\delta_{\text {max }}=100^{\circ}$, the steering wheel angle does not reach it since is not needed to turn that much to perform the maneuver. Whereas, for the case of the lateral controller, the steering is jerky especially at the beginning where is discontinuous.

In figs. 9 to 11 , is shown the yaw-rate response for the cases when the RG is out of the loop, and when it is used with $\delta_{\max }=50^{\circ}, 100^{\circ}$ respectively. In Fig. 9, the RG is absent and the yaw-rate of the vehicle overshoots during the tracking of the reference which is discontinuous at the beginning. On the other hand, the virtual reference shown in figs. 10 and 11 is gentler compared to the desired one and for that reason the steering wheel angle is increasing in an admissible rate. In Fig. 10, the virtual reference saturates automatically to a certain value so as to avoid the violation of the steering wheel constraint.

\section{CONCLUSIONS \& FUTURE WORK}

This paper proposes a RG approach to merge lane change and lane tracking for a yaw-rate tracking system. The lane change relies on the tracking of a virtual reference, enabling a fast controller to track an adjacent lane. The virtual reference is smooth and permits the convergence to the desired lane without oscillations.

Future works include the experimental validation of the proposed methodology on the automated Renault Zoe.

\section{ACKNOWLEDGMENT}

This paper reflects solely the views of the authors and not necessarily the view of the company they belong to. 


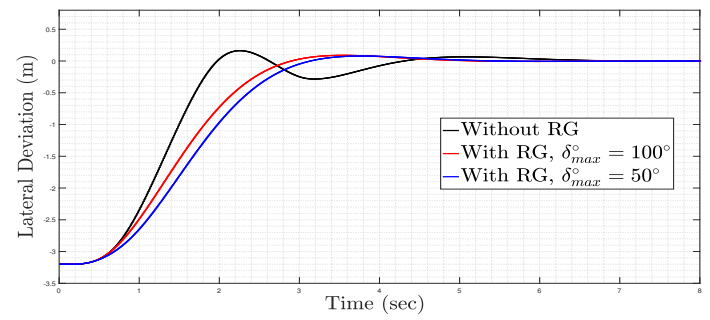

Fig. 7. Lateral deviation during lane-change. In blue is the response when $\delta_{\max }^{\circ}=50$, in red when $\delta_{\max }^{\circ}=100$ and in black when the RG is absent.

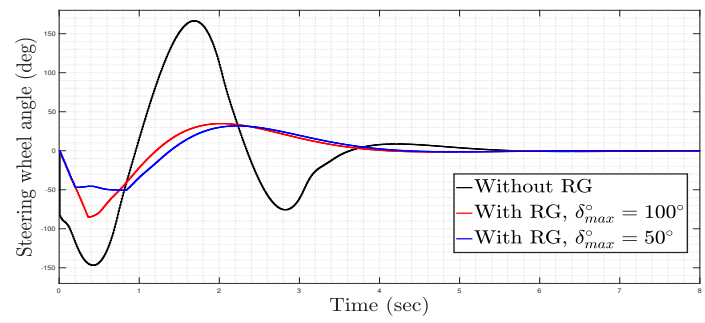

Fig. 8. Steering wheel angle during lane-change. In blue is the response when $\delta_{\max }^{\circ}=50$, in red for $\delta_{\max }^{\circ}=100$ and in black when the RG is absent.

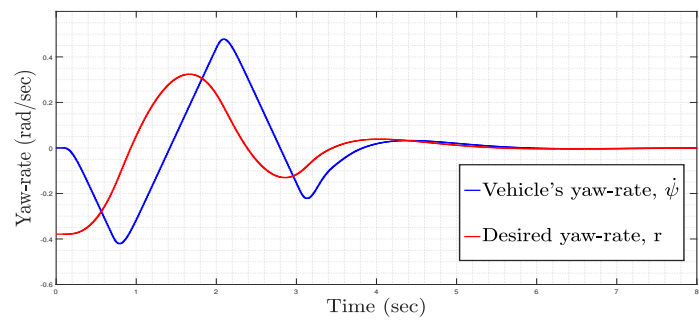

Fig. 9. Yaw-rate references during lane-change without RG. In blue is depicted the vehicle's yaw rate and in red the desired yaw-rate.

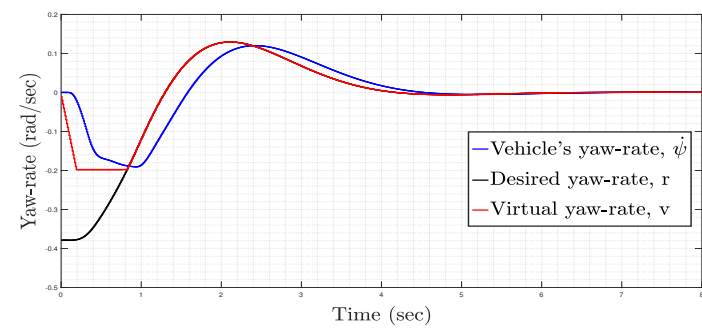

Fig. 10. Yaw-rate references during lane-change when the RG is utilized and $\delta_{\max }^{\circ}=50$. Vehicle's yaw rate is in blue, the desired in black and the virtual in red.

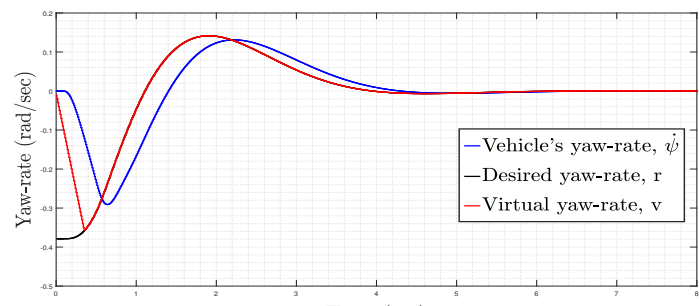

Time (sec)

Fig. 11. Yaw-rate references during lane-change when the RG is utilized and $\delta_{\max }^{\circ}=100$. Vehicle's yaw rate is in blue, the desired in black and the virtual in red.

\section{REFERENCES}

[1] D. González, J. Pérez, V. Milanés, and F. Nashashibi, "A review of motion planning techniques for automated vehicles," IEEE Transactions on Intelligent Transportation Systems, vol. 17, no. 4, pp. 1135-1145, 2015.

[2] D. Kapsalis, O. Sename, V. Milanés, and J. J. Martinez, "Gainscheduled steering control for autonomous vehicles," IET Control Theory \& Applications, 2020.

[3] H.-S. Tan and J. Huang, "Design of a high-performance automatic steering controller for bus revenue service based on how drivers steer," IEEE Transactions on Robotics, vol. 30, no. 5, pp. 1137-1147, 2014.

[4] P. Falcone, F. Borrelli, J. Asgari, H. E. Tseng, and D. Hrovat, "Predictive active steering control for autonomous vehicle systems," IEEE Transactions on control systems technology, vol. 15, no. 3, pp. 566-580, 2007.

[5] D. Bevly, X. Cao, M. Gordon, G. Ozbilgin, D. Kari, B. Nelson, J. Woodruff, M. Barth, C. Murray, A. Kurt, et al., "Lane change and merge maneuvers for connected and automated vehicles: A survey," IEEE Transactions on Intelligent Vehicles, vol. 1, no. 1, pp. 105-120, 2016.

[6] E. Garone, S. Di Cairano, and I. Kolmanovsky, "Reference and command governors for systems with constraints: A survey on theory and applications," Automatica, vol. 75, pp. 306-328, 2017.

[7] E. G. Gilbert, I. Kolmanovsky, and K. T. Tan, "Discrete-time reference governors and the nonlinear control of systems with state and control constraints," International Journal of robust and nonlinear control, vol. 5, no. 5, pp. 487-504, 1995.

[8] E. G. Gilbert and K. T. Tan, "Linear systems with state and control constraints: The theory and application of maximal output admissible sets," IEEE Transactions on Automatic control, vol. 36, no. 9, pp. $1008-1020,1991$

[9] A. Bemporad, A. Casavola, and E. Mosca, "Nonlinear control of constrained linear systems via predictive reference management," IEEE transactions on Automatic Control, vol. 42, no. 3, pp. 340-349, 1997.

[10] I. Kolmanovsky, E. Garone, and S. Di Cairano, "Reference and command governors: A tutorial on their theory and automotive applications," in 2014 American Control Conference. IEEE, 2014, pp. 226-241.

[11] R. Bencatel, R. Tian, A. R. Girard, and I. Kolmanovsky, "Reference governor strategies for vehicle rollover avoidance," IEEE Transactions on Control Systems Technology, vol. 26, no. 6, pp. 1954-1969, 2017.

[12] B. Sakhdari and N. L. Azad, "A distributed reference governor approach to ecological cooperative adaptive cruise control," IEEE Transactions on Intelligent Transportation Systems, vol. 19, no. 5, pp. 1496-1507, 2017.

[13] I. Mahtout, F. Navas, D. Gonzalez, V. Milanés, and F. Nashashibi, "Youla-kucera based lateral controller for autonomous vehicle," in 2018 21st International Conference on Intelligent Transportation Systems (ITSC). IEEE, 2018, pp. 3281-3286.

[14] R. Rajamani, Vehicle dynamics and control. Springer Science \& Business Media, 2011.

[15] S. Skogestad and I. Postlethwaite, Multivariable Feedback Control. Analysis and Design. John Wiley and Sons, Chichester, 2005.

[16] P. Gahinet and P. Apkarian, "A linear matrix inequality approach to $h_{\infty}$ control," International journal of robust and nonlinear control, vol. 4 , no. 4, pp. 421-448, 1994.

[17] M. Herceg, M. Kvasnica, C. Jones, and M. Morari, "MultiParametric Toolbox 3.0," in Proc. of the European Control Conference, Zürich, Switzerland, July 17-19 2013, pp. 502-510, http://control.ee.ethz.ch/ mpt.

[18] J. Mattingley and S. Boyd, "Cvxgen: A code generator for embedded convex optimization," Optimization and Engineering, vol. 13, no. 1, pp. 1-27, 2012. 